\title{
Analysis of marketing data to extract key factors of telecom churn management
}

\author{
Hao-En Chueh \\ Department of Information Management, Yuanpei University, No. 306, Yuanpei Street, Hsienchu 30015, Taiwan, \\ R. O. C. E-mail: hechueh@mail.ypu.edu.tw. Tel: +886-3-5381183/8292. Fax: +886-3-6102362. \\ Accepted 16 May, 2011

\begin{abstract}
For telecom industries facing customer and market changes due to rapid developments, near saturation, and intense competition, avoiding churn is critical. Most churn management studies attempt to locate a list of potentially lost customers from existing customer data, for use in subsequent customer retention activities. However, the effectiveness of a telecom company's churn management is determined by whether it decreases the churn rate and successfully retains lost customers. To help telecom industries achieve effective churn management, this study utilizes fuzzy correlation analysis to extract the key factors of telecom churn management processes. Using a dataset of a telecom company in Taiwan, a data mining-based churn management model was constructed in previous work. The key factors identified by the data mining-based churn management model are confirmed by fuzzy correlation analysis.
\end{abstract}

Key words: Telecom industry, churn management, fuzzy correlation analysis, data mining, customer retention strategy.

\section{INTRODUCTION}

When an industry's customer market transitions from rapid development to near saturation and intense competition, that industry faces severe churn problems. Examples of this scenario include telecom industries, internet services industries, banking-related industries, and even the cultural and education industries (Ahn et al., 2006; Buckinx et al., 2005; Keaveney, 1995; Kim et al., 2004; Kim et al., 2004). Numerous enterprises have entered the telecom industry to acquire a portion of the emerging telecom market ever since the government fully liberalized the mobile phone industry. Initially, there was a massive demand for emerging telecom services because mobile phone service users were uncommon. As a result, the mobile telecom industry developed rapidly.

However, since the demand for mobile telecom services has transitioned from rapid growth to near saturation in recent years, large telecom companies have become intensely competitive. Compounding this situation is the Taiwan government's introduction of portable mobile numbers, allowing users to switch mobile phone carriers without changing their mobile phone numbers. Users can easily switch their mobile phone service carriers to maximize their benefits, meaning, telecom companies could face serious churn problems (Ahn et al.,
2006; Kim et al., 2004; Kim et al., 2004; Mattersion, 2001).

Nowadays, mobile telecom services have become an indispensable communication channel. Since everyone requires mobile telecom services, customers lost by company $A$ will inevitably become customers of company $B$ or company $C$. Previous studies indicate that to gain a new customer, a company must spend five to ten times the amount needed to retain a customer. Therefore, retaining long-term loyal customers is more profitable than gaining new short-term customers. To maintain market share and profitability, telecom companies have used various approaches or management mechanisms to retain customers and prevent serious churn problems. Maintaining existing customers, locating potentially lost customers in advance, and effectively implementing customer retention strategies, are all serious concerns faced by telecom companies (Mattersion, 2001).

A common churn management process involves constructing a churn prediction model using past churn data, and determining key factors affecting churn. This churn model is then used to locate a list of potentially lost customers from existing customer data, to perform retention activities (Berson et al., 2000; Chu et al., 2007; 
Hung et al., 2007; Lariviere et al., 2004; Mozer et al., 2000; Ngai et al., 2009). The success of a company's churn management is determined by whether it effectively decreases churn rate, and not by whether it can locate a list of potentially lost customers. The critical element of churn management is successful retention of potentially lost customers. However, most telecom companies underestimate the importance of effective customer retention strategies. Consequently, this study utilizes fuzzy correlation analysis (Chiang et al., 1999; Chiang et al., 2000) to analyze the results of marketing activities to extract the key factors of telecom churn management.

This paper is organized as follows: Section 2 presents several studies on telecom churn management. Section 3 introduces the analysis method, fuzzy correlation analysis (Chiang et al., 1999; Chiang et al., 2000), to extract the key factors of telecom churn management. Section 4 presents the experimental results, while Section 5 provides the conclusions.

\section{TELECOM CHURN MANAGEMENT}

When telecom companies face churn problems, they implement feasible churn management procedures and strengthen existing customer relationship management. Previous studies utilized various data mining technologies to assist telecom companies in resolving churn problems (Coussement et al., 2008; Hung et al. 2006; Kim et al., 2004; Kim et al., 2004; Mozer et al., 2000; Tsai et al., 2010; Tsai et al., 2009; Wei et al., 2002; Xia et al. 2008).

Data mining refers to using automatic or semiautomatic methods to extract latent, unknown, meaningful, and useful information or models from large datasets (Berry et al., 2004; Dunham, 2003; Fayyad et al., 1996; Han et al., 2001; Kantardzic, 2003; Tan et al., 2006). Further discussion describes the main techniques involved in this process.

Classification analysis classifies a new example without a clear category into a predetermined category. This technique is broadly applied to resolve problems in different fields, for example, using document titles and content for classification, and using various medical test results to determine whether tumors are malignant or benign. Common classification analysis methods include decision trees, nearest neighbor classification, Bayesian classification, neural networks, and support vector machines.

Prediction analysis utilizes previously or currently recorded information or conditions to determine possible future results. Most methods applicable to classification analysis can also be applied to predictive models. The difference between these approaches is that classification analysis is used to analyze current conditions, while prediction analysis is used to predict future conditions.

Clustering analysis produces highly similar cluster combinations by analyzing the similarities between numerous examples or records. The primary purpose of clustering degrees of similarity with examples or records within other clusters. More discriminatory cluster sets are produced when there is high similarity within clusters and high variation between clusters.

Association rule analysis locates the rules of specific associations from data. The most popular association rule is dependency relationships in item sets of databases. Association rules established using similarity relationships can exhibit sets of items that appear simultaneously in databases.

Sequential pattern analysis is a technique similar to association rule analysis; however, it differs by emphasizing dependence in item sets and incorporating temporal sequences. The primary purpose of sequential pattern analysis is locating the temporal sequence relationships of item sets that commonly appear in a database within a specific time interval.

Data mining is an integrated technique that involves analysis, filtering, extraction, and statistical analysis for large amounts of data; it has numerous applications in addressing business problems (Au et al., 2003; Berson et al., 2000; Chu et al., 2007; Coussement et al., 2008; Lariviere et al., 2004; Lejeune, 2001; Luo et al., 2007; Ngai et al., 2009).

Wei et al. (2002) constructed a churn prediction model that identifies churners from subscriber contractual information and call pattern changes from call details. This study proposes a multi-classifier class-combiner approach to address the challenge of a highly skewed class distribution between churners and non-churners. The proposed approach achieves satisfactory or reasonable prediction within the one-month interval between model construction and churn prediction.

Hung et al. (2006) proposed the use of customer demography data, bill payment information, call detail records, customer care/service status, and service change logs to indentify potentially lost customers, using data mining techniques. This study compares various data mining techniques capable of assigning a churn score to each mobile subscriber. Results indicate that both the decision tree and neural network methods can deliver accurate churn prediction models.Xia et al. (2008) proposed a SVM-based customer churn prediction model to improve prediction abilities by applying structural risk minimization. This study compares the proposed method with artificial neural network, decision tree, logistic regression, and naive Bayesian classifier methods, demonstrating that the proposed method outperforms other methods regarding accuracy rate, hit rate, covering rate, and lift coefficient. Coussement et al. (2008) established a decision support system for telecom churn prediction. This study evaluates the benefits of adding the voice of the customers through call center e-mails (textual information) to a conventional churn prediction system using only traditional marketing information. These results show a significant increase in predictive 
performance by adding unstructured, textual information to a conventional churn prediction system. From a managerial perspective, this information can enable decisionmakers to identify customers most prone to switch.

Tsai et al. (2009) adopted hybrid neural network based models to manage telecom churn problems. This study constructs two hybrid models by combining two different neural network techniques for churn prediction. The first model is based on back propagation artificial neural networks (ANNs) and self-organizing maps (SOMs). The second model is the first model combined with ANN, in other words, ANN + ANN. Experimental results show that the two hybrid models outperform the single neural network baseline model regarding prediction accuracy. The ANN + ANN hybrid model performs better than the ANN + SOM hybrid model significantly.

The purpose of the studies reviewed is constructing an effective churn predictive model to determine a list of potentially lost customers, providing telecom companies with a reference for implementing customer retention activities. However, for telecom customers, locating potentially lost customers does not equate to retaining customers and effectively reducing churn rates. Therefore, this study emphasizes framing an effective customer retention strategy to help telecom companies effectively reduce churn rates. This study utilizes fuzzy correlation analysis (Chiang et al., 1999; Chiang et al., 2000) to analyze the responses of different customer groups to retention activities to identify the key factors of telecom churn management and determine an effective customer retention strategy. This approach provides telecom companies with a reference to determine optimal retention timing and retention method combinations for different customer groups.

\section{MATERIALS AND METHODS}

\section{Fuzzy correlation analysis}

This study emphasizes extracting the key factors of telecom churn management, enabling telecom companies to determine the most appropriate retention timing, and retention method combinations for different customer groups. Therefore, a fuzzy correlation analysis (Chiang et al., 1999; Chiang et al., 2000) is used.

Researchers frequently use correlation analyses to determine the relationships between the attributes of databases (Dowdy et al., 1983). Conventional statistics have comprehensively discussed the various correlation analyses defined on ordinary crisp sets. However, attributes recorded in databases may be fuzzy (Zadeh, 1965) but important, and are waiting to be explored. Therefore, methods to investigate these fuzzy attributes are required. This section introduces fuzzy simple correlation analysis (Chiang et al., 1999) on Zadeh's fuzzy sets (Zadeh, 1965). The simple correlation coefficient between two fuzzy sets is referred to as the fuzzy simple correlation coefficient (Chiang et al., 1999).

Suppose there are two fuzzy sets $A, B \subset F$, where $F$ is a fuzzy space, the fuzzy set $A$ and $B$ are defined on a crisp universal set $X$ with membership functions $\mu_{A}$ and $\mu_{B}$. Then the fuzzy sets $A$ and $B$ can be expressed as:

$$
\begin{aligned}
& \left.A=\left(x, \mu_{A}(x)\right) \mid x \in X\right), \\
& \left.B=\left(x, \mu_{B}(x)\right) \mid x \in X\right),
\end{aligned}
$$

where $\mu_{A}: X \rightarrow[0,1]_{\text {and }} \mu_{B}: X \rightarrow[0,1]$.

Assuming there is a random sample $\left(x_{1}, x_{2}, \cdots, x_{n}\right) \in X$, alone with a sequence of paired data,

$\left(\left(x_{1}, \mu_{A}\left(x_{1}\right), \mu_{B}\left(x_{1}\right)\right),\left(x_{2}, \mu_{A}\left(x_{2}\right), \mu_{B}\left(x_{2}\right)\right), \cdots,\left(x_{n}, \mu_{A}\left(x_{n}\right)\right.\right.$, $\left.\mu_{B}\left(x_{n}\right)\right)$ ), which correspond to the grades of the membership functions of fuzzy sets $A$ and $B$ defined on $X$.

Then, the simple correlation coefficient between the fuzzy sets $A$ and $B, r_{A, B}$ is as follows (Chiang et al., 1999; Dowdy et al., 1983):

$r_{A, B}=\frac{\sum_{i=1}^{n}\left(\mu_{A}\left(x_{i}\right)-\bar{\mu}_{A}\right)\left(\mu_{B}\left(x_{i}\right)-\bar{\mu}_{B}\right) /(n-1)}{S_{A} \cdot S_{B}}$

$\bar{\mu}_{A}=\frac{\sum_{i=1}^{n} \mu_{A}\left(x_{i}\right)}{n}$

$\bar{\mu}_{B}=\frac{\sum_{i=1}^{n} \mu_{B}\left(x_{i}\right)}{n}$

$S_{A}^{2}=\frac{\sum_{i=1}^{n}\left(\mu_{A}\left(x_{i}\right)-\bar{\mu}_{A}\right)^{2}}{n-1}$

$S_{B}^{2}=\frac{\sum_{i=1}^{n}\left(\mu_{B}\left(x_{i}\right)-\bar{\mu}_{B}\right)^{2}}{n-1}$

$S_{A}=\sqrt{S_{A}^{2}}$

$S_{B}=\sqrt{S_{B}^{2}}$

where $\bar{\mu}_{A \text { and }} \bar{\mu}_{B}$ denote the average membership grades of fuzzy sets $A$ and $B$ over the random sample, $S_{A \text { and }} S_{B}$ are the sample standard deviations of fuzzy sets $A$ and $B$, respectively.

Several important properties of the fuzzy simple correlation 
coefficient can be obtained and stated as follows:

If $\left|r_{A, B}\right|$ is close to 1 , then the fuzzy sets $A$ and $B$ are highly related.

If $\left|r_{A, B}\right|$ is close to 0 , then the fuzzy sets $A$ and $B$ are barely related.

If $r_{A, B}>0$, then the fuzzy sets $A$ and $B$ are positively related.

If $r_{A, B}<0$, then the fuzzy sets $A$ and $B$ are negatively related.

If $r_{A, B}=0$, then the fuzzy sets $A$ and $B$ have no relationship at all.

Though the values of the fuzzy membership function are constrained between in $[0,1]$, the value of the fuzzy simple correlation coefficient lies between in $[-1,1]$. This not only reveals the degree of the relationship between the fuzzy sets, but also indicates whether these two sets are positively or negatively related.

\section{Key factors of telecom churn management}

\section{Experimental setting}

The experimental dataset used in this study resulted from the randomly sampled customer retention activities and the responses of customers of a telecom company in Taiwan whose contracts were due to expire between June and July 2008. The company developed two marketing programs to retain their customers. The first program involved a discount on monthly bills, while the second program was a cellular phone purchase promotion. From the customers whose contracts were due to expire in June and in July 2008, 400 customers were randomly selected from each of the following groups: customers with monthly bills of NT\$ 0 to 300 , customers with monthly bills of NT\$301 to 800, and customers with monthly bills of NT\$ 801 to 1000 . Each group of 400 customers were then divided further into two subgroups of 200 customers each. Customer retention marketing programs were implemented using direct mail (indirect marketing) and telemarketing (direct marketing). During this retention marketing process, customers could choose the marketing programs they wanted. Table 1 displays the results of the entire retention marketing process.

\section{ANALYSES AND RESULTS}

To extract the key factors of telecom churn management, this study uses a simple fuzzy correlation coefficient to analyze the correlation relationship between retention rate and other attributes in Table 1. The attributes in Table 1 must be converted into fuzzy attributes. $\mu_{C V P}$ is the membership function of contract validity period; $\mu_{B P}$ is the membership function of bill payment; $\mu_{M M}$ is the membership function of marketing method; while $\mu_{R R}$ is the membership function of retention rate. $\mu_{C V P}, \mu_{B P}$, $\mu_{M M}, \mu_{R R}$ are shown as follows:

$$
\mu_{C V P}(x)=\left\{\begin{array}{cc}
0 & , x=0 \\
0.2 & , x=1 \\
0.5 & , x=2 \\
1 & , x>2
\end{array}\right.
$$

$$
\begin{aligned}
& \mu_{B P}(x)=\left\{\begin{array}{cl}
0 & , x=0 \\
0.3 & , x=0 \sim 300 \\
0.7 & , x=301 \sim 800 \\
1 & , x>801
\end{array}\right. \\
& \mu_{M M}(x)=\left\{\begin{array}{cl}
0.5 & , x=\text { indirect marketing } \\
1 & , x=\text { direct marketing }
\end{array}\right.
\end{aligned}
$$

$$
\mu_{R R}(x)=\left\{\begin{array}{cl}
0 & , x \leq 0 \\
0.2 & , 0<x \leq 0.2 \\
0.4 & , 0.2<x \leq 0.4 \\
0.6 & , 0.4<x \leq 0.6 \\
0.8 & , 0.6<x \leq 0.8 \\
1.0 & , 0.8<x
\end{array}\right.
$$

According to Equation (3), the fuzzy correlation coefficient between retention rate and contract validity period, $r_{R R, C V P}$, is equal to 0.06 ; the fuzzy correlation coefficient between retention rate and bill payment, $r_{R R, B P}$, is equal to 0.38 ; the fuzzy correlation coefficient between retention rate and marketing method, $r_{R R, M M}$, is equal to 0.57 . Therefore, the most important factor of churn management is marketing method.

Previous research (Chueh et al., 2011) used the decision tree technique (Berry et al., 2004; Dunham, 2003; Fayyad et al., 1996; Han et al., 2001; Kantardzic, 2003; Quinlan, 1986; Tan et al., 2006) to construct an effective churn management model and reduce churn rates based on customer responses to retention activities performed by customer service centers. Figure 1 shows the proposed customer retention model.

According to Figure 1, the churn retention model confirms the key factors determined by fuzzy correlation analysis. The customer retention model indicates that, for customers with monthly bills of NT\$301 to 800 and NT\$801 to 1000, telemarketing was an effective customer retention strategy for customers whose contracts expired in either June or July. However, for customers whose monthly bills were NT\$0 to 300 and whose contracts 
Table 1. The results of customer retention activities.

\begin{tabular}{ccclc}
\hline Customer group & Contract validity period (month) & Bill payment (NT\$) & Marketing method & Retention rate (\%) \\
\hline 1 & 1 & $0-300$ & Direct marketing & 17 \\
2 & 1 & $0-300$ & Indirect marketing & 25 \\
3 & 1 & $301-800$ & Direct marketing & 48 \\
4 & 1 & $301-800$ & Indirect marketing & 4 \\
5 & 1 & $801-1000$ & Direct marketing & 87 \\
6 & 1 & $801-1000$ & Indirect marketing & 13 \\
7 & 2 & $0-300$ & Direct marketing & 18 \\
8 & 2 & $0-300$ & Indirect marketing & 22 \\
9 & 2 & $301-800$ & Direct marketing & 48 \\
10 & 2 & $301-800$ & Indirect marketing & 4 \\
11 & 2 & $801-1000$ & Direct marketing & 74 \\
12 & 2 & $801-1000$ & Indirect marketing & 11 \\
\hline
\end{tabular}

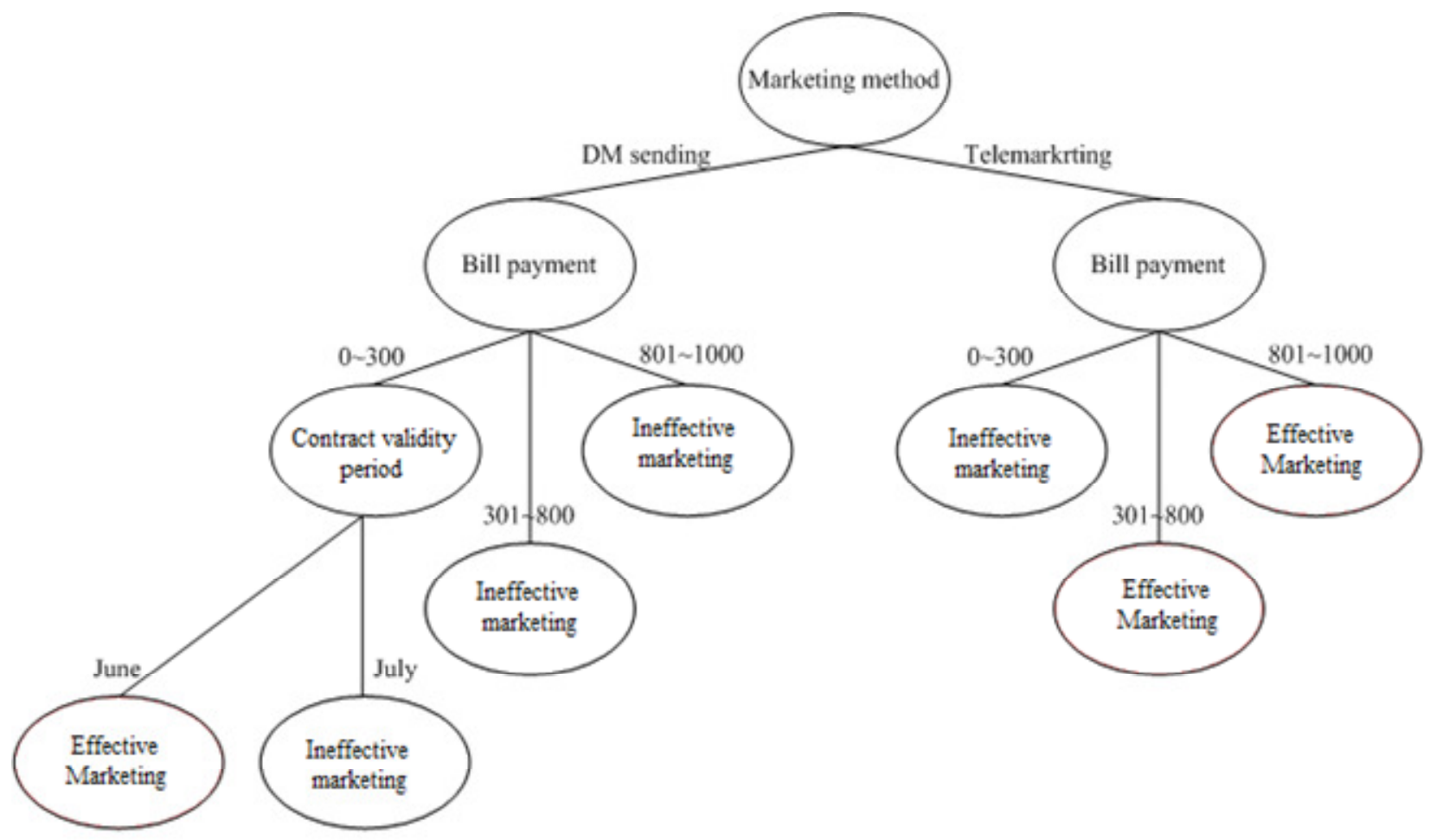

Figure 1. Telecom churn management model.

expired in June, only a direct mail sent in June (the contract expiration month) was an effective customer retention strategy.

Table 1 also indicates that for customers whose monthly bills were NT\$ 801 to 1000 and whose contracts expired in June, telemarketing in June was the most effective customer retention strategy. The customer retention rate for this customer group was $87 \%$. However, for customers whose monthly bills were NT\$ 801 to 1000 and whose contracts expired in July, telemarketing performed in June (one month before contract expiration) achieved a $74 \%$ customer retention rate, $13 \%$ lower than the best customer group. Telemarketing performed during the month of contract expiration had the greatest effect on customer retention.

\section{Conclusion}

Most studies of the telecom churn problem focus on 
constructing an effective churn prediction model to locate lists of potentially lost customers in advance. However, identifying potentially lost customers does not mean those potentially lost customers can be retained. An effective customer retention strategy must be employed to effectively reduce churn rates. This study utilized fuzzy statistics analysis to identify the key factors of telecom churn management. These key factors were confirmed by a data mining-based churn management model in previous research. Analysis of customer responses to marketing activities is useful in determining optimal retention timing and retention method combinations when developing effective customer retention strategies for different customer groups.

\section{REFERENCES}

Ahn JH, Han SP, Lee YS (2006). Customer churn analysis: Churn determinants and mediation effects of partial defection in the Korean mobile telecommunications service industry. Telecomm. Policy, 30: 552-568.

Au W, Chen KCC, Yao X (2003). A novel evolutionary data mining algorithm with applications to churn prediction. IEEE Trans. Evol. Comput., 7: 532-545.

Berry MJA, Linoff G. (2004). Data mining techniques: For marketing, sales, and customer support. NY: John Wiley \& Sons.

Berson A, Simith S, Thearling K (2000). Building data mining applications for CRM, NY: McGraw-Hill.

Buckinx W, Van den Poel D (2005). Customer base analysis: partial defection of behaviourally loyal clients in a non-contractual FMCG retail setting. Eur. J. Oper. Res., 164: 252-268.

Chu BH, Tsai MS, Ho CS (2007). Toward a hybrid data mining model for customer retention. Knowl. Based Syst., 20: 703-718.

Chiang DA, Lin NP (1999). Correlation of Fuzzy Sets. Fuzzy Sets Syst., 102: $221-226$

Chiang DA, Lin NP (2000). Partial Correlation of Fuzzy Sets. Fuzzy Sets Syst., 110: 209-215.

Chueh HE, Lin SC, Jan NY (2011). Mining the Telecom Marketing Information to Optimizing the Customer Retention Strategies. ICIC Express Lett. Part B Appl., 2: 1-6.

Coussement K, Van den Poel D (2008). Churn prediction in subscription services: An application of support vector machines while comparing two parameter-selection techniques. Expert. Syst. Appl., 34: 313-327.

Coussement K, Van den Poel D (2008). Integrating the voice of customers through call center emails into a decision support system for churn prediction. Inform. Manage., 45: 164-174.

Dowdy S, Wearden S (1983). Statistics for research. John Wiley and Sons, NY.

Dunham MH (2003). Data mining, introductory and advanced topics. $\mathrm{NJ}$ : Pearson Education.

Fayyad U, Uthurusamy R (1996). Data mining and knowledge discovery in databases. Commun., ACM. 39: 24-27.
Han J, Kamber M (2001). Data Mining: Concepts and Techniques. NY: Morgan Kaufmann.

Hung SY, Yen DC, Wang HY (2006). Applying data mining to telecom churn management. Expert. Syst. Appl., 31:515-524.

Kantardzic M (2003). Data mining-Concepts, models, methods, and algorithms. John Wiley \& Sons, NY.

Keaveney SM (1995). Customer switching behavior in service industries: An exploratory study. J. Mark., 59: 71-82.

Kim HS, Yoon CH (2004). Determinants of subscriber churn and customer loyalty in the Korean mobile telephony market. Telecomm. Policy, 28: 751-765.

Kim MK, Park MC, Jeong DH (2004). The effects of customer satisfaction and switching barrier on customer loyalty in Korean mobile telecommunication services. Telecomm. Policy, 28: 145-159.

Lariviere B, Van den Poel D (2004). Investigating the role of product features in preventing customer churn, by using survival analysis and choice modeling: The case of financial services. Expert. Syst. Appl., 27: 277-285.

Lejeune $M$ (2001). Measuring the impact of data mining on churn management, Internet Research. Electr. Network Appl. Policy, 11: 375-387.

Luo B, Shao P, Liu D (2007). Evaluation of three discrete methods on customer churn model based on neural network and decision tree in PHSS. Proceedings of the first international symposium on data privacy, and e-commerce, 95-97.

Mattersion R (2001). Telecom churn management. NC: APDG Publishing.

Mozer MC, Wolniewicz R, Grimes DB, Johnson E, Kaushanky H (2000). Predicting subscriber dissatisfaction and improving retention in the wireless telecommunications industry. IEEE T. Neural Netw., 11: 690696.

Ngai EWT, Xiu L, Chau DCK (2009). Application of data mining techniques in customer relationship management: A literature review and classification. Expert. Syst. Appl., 36: 2592-2602.

Quinlan JR (1986). Induction of Decision Trees. Mach. Learn. 1: 81 106.

Tan PN, Steinbach M, Kumar V (2006). Introduction to data mining, Boston: Pearson Addison Wesley.

Tsai CF, Chen MY (2010). Variable selection by association rules for customer churn prediction of multimedia on demand. Expert. Syst. Appl., 37: 2006-2015.

Tsai CF, Lu YH (2009). Customer churn prediction by hybrid neural networks. Expert. Syst. Appl., 36: 12547-12553.

Wei CP, Chiu IT (2002).Turning telecommunications call details to churn prediction: a data mining approach. Expert. Syst. Appl., 23: 103-112.

Xia GE, Jin WD (2008). Model of customer churn prediction on support vector machine. Syst. Eng. Theory Pract., 28: 71-77.

Zadeh LA (1965). Fuzzy sets. Inform. Control, 8: 338-353. 